\title{
Ecualizador adaptativo multi-rate en tiempo continuo para comunicaciones serie de alta velocidad
}

\author{
Erick Guerrero, Cecilia Gimeno, Carlos Sánchez, Javier Aguirre, Santiago Celma \\ Grupo de Diseño Electrónico (GDE) \\ Instituto de Investigación en Ingeniería de Aragón (I3A). \\ Universidad de Zaragoza, Mariano Esquillor s/n, 50018, Zaragoza, Spain. \\ Tel.+34-976762707, Fax +34-976762043, e-mail: eguerrero@unizar.es
}

\begin{abstract}
Se presenta un nuevo concepto de ecualización adaptiva multi-rate para comunicaciones serie de alta velocidad. Ha sido comprobado su correcto funcionamiento en transmisión por fibra óptica plástica (POF) de hasta $50 \mathrm{~m}$ para datos de entrada desde 400 Mbps hasta 1.25 Gbps. El circuito puede operar con una tensión de alimentación de sólo $1 \mathrm{~V}$ y un consumo menor a $39 \mathrm{~mW}$.
\end{abstract}

\section{Introducción}

La demanda de mayores tasas de transmisión para aplicaciones en el hogar ha incrementado los esfuerzos para desarrollar sistemas de comunicación de bajo coste, confiables y fáciles de instalar. La fibra óptica plástica (POF) es una buena opción como medio de transmisión para lograr este objetivo debido su ligereza, robustez y fácil instalación. Sin embargo, debido a que su ancho de banda es muy limitado, fenómenos no deseados tales como la interferencia inter-símbolo (ISI), pueden causar errores en los datos recibidos. Para corregir los errores en el proceso de comunicación a tasas de transmisión superiores al gigabit por segundo, es preferible la ecualización de la señal en tiempo continuo sobre otras técnicas de ecualización más complejas basadas en criterios de adaptación digitales. Adicionalmente, debido a las variaciones en las características del canal por temperatura, longitud, curvatura, etc., el ecualizador debe ser capaz de adaptar su respuesta para compensar dichas variaciones.

La técnica de balanceo espectral, cuyo esquema conceptual se muestra en la Figura 1, es la mejor opción para lograr un sistema de baja complejidad y bajo consumo de potencia, sobre otras propuestas en la literatura. Su principio de operación se basa en la forma $\operatorname{sinc}^{2}$ que tiene la densidad espectral de potencia (PSD) de las señales NRZ idealmente aleatorias. $\mathrm{Si}$ podemos comparar la razón de potencias entre dos rangos de frecuencia distintos, se puede determinar si la señal está idealmente, sub o sobre-compensada.

\section{Diseño del circuito}

Para la función $\operatorname{sinc}^{2}$, la razón de dos potencias de señales, $P_{1}$ y $P_{2}$, para 2 rangos de frecuencia cualesquiera, $f_{1}$ y $f_{2}$, es constante y se puede expresar como

$$
\frac{P_{2}}{P_{1}}=C
$$

donde las potencias $P_{1}$ y $P_{2}$ se pueden obtener mediante dos filtros pasa-bajas LPF1 y LPF2, respectivamente. Por lo tanto, la señal de control se puede generar a partir de la desviación de la condición (1)

$$
V_{C} \approx P_{1} \cdot c-P_{2}
$$

Sin embargo, si las frecuencias de corte se mantienen constantes para cambios en la tasa de transferencia de la señal, el valor de $c$ será desplazado provocando errores en la comparación de potencias. Este problema se ilustra en la Figura 2; cuando la tasa de bit cambia de 1.25 Gbps a 400 Mbps, el lóbulo principal contiene ahora todo el ancho de banda del filtro LPF2. Esto incrementa la potencia $P_{2}$, y ahora $V_{C}$ se hace más pequeña. Por lo tanto, es preciso desplazar la frecuencia desde $f_{2 a}$ hasta $f_{2 \mathrm{~b}}$, para la nueva tasa de bits. La Figura 3 muestra la arquitectura del ecualizador adaptativo multi-rate propuesto; estructura mejorada de [1]. El lazo de adaptación de la izquierda realiza una comparación de las potencias $P_{1}$ y $P_{2}$, y genera un voltaje de control, $V_{C}$, para ajustar la ganancia [2]. La frecuencia de corte del LPF2 es digitalmente programable mediante una palabra digital, $D$, la cual selecciona una combinación de condensadores en paralelo. La Tabla 1 presenta las diferentes frecuencias de corte disponibles, las cuales han sido elegidas de tal forma que se obtenga un error cero para una señal NRZ ideal, para cuatro tasas de transmisión desde 400 Mbps hasta 1.25 Gbps. Las 
ganancias de ambos filtros, LPF1 y LPF2, son de 6

\begin{tabular}{|c|c|c|c|}
\hline Input data rate & $D_{2} D_{1} D_{0}$ & BW $_{L P F 2}$ & BW $_{L P F 1}$ \\
\hline 1.25 Gbps & 000 & $400 \mathrm{MHz}$ & \multirow{4}{*}{$30 \mathrm{MHz}$} \\
\hline 970 Mbps & 001 & $309 \mathrm{MHz}$ & \\
\hline $680 \mathrm{Mbps}$ & 011 & $212 \mathrm{MHz}$ & \\
\hline $400 \mathrm{Mbps}$ & 111 & $128 \mathrm{MHz}$ & \\
\hline
\end{tabular}

Tabla 1. Frecuencias de corte Dara LPF1 v LPF2

dB y $3.5 \mathrm{~dB}$, respectivamente.

El comparador o slicer en la salida del ecualizador restaura la señal a una secuencia binaria con una PSD con forma $\operatorname{sinc}^{2}$ bien definida. Los cambios en la tasa de transmisión causarán un desplazamiento del primer cero, $1 / T_{B}$, lo que incrementa 0 disminuye la potencia de alta frecuencia. Estos cambios son detectados por el comparador de potencia en el segundo lazo, y se genera la señal digital de control para ajustar la frecuencia de corte del LPF2.

\section{Resultados de simulación}

La trama de test es una secuencia pseudo aleatoria de longitud $2^{31}-1$ modulada NRZ y afectada por la respuesta frecuencial de una SI-POF Mitsubishi HG. El ecualizador propuesto ha sido diseñado en una

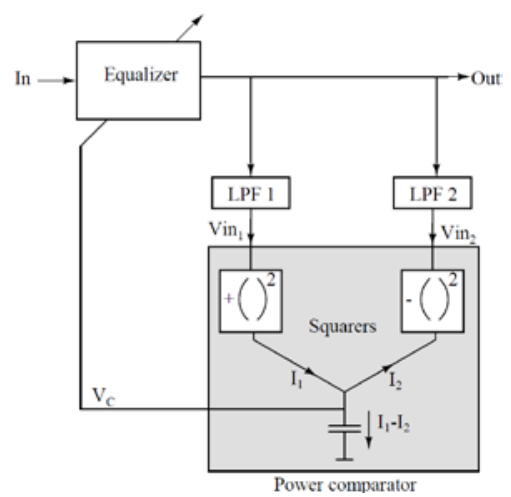

Figura 1. Esquema conceptual de ecualización basada en la técnica de balanceo espectral

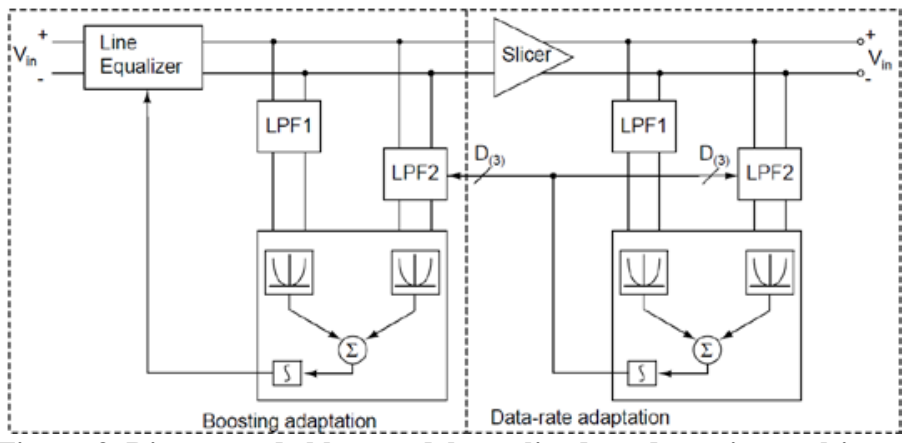

Figura 3. Diagrama de bloques del ecualizador adaptativo multi-rate propuesto tecnología estándar CMOS de $0.18 \mu \mathrm{m}$ y está alimentado con una tensión de $1 \mathrm{~V}$. Las Figuras 4 y 5 muestran los diagramas de ojos (a) antes y (b) después de la ecualización de la señal transmitida sobre una fibra de $50 \mathrm{~m}$ de longitud, para tasas de bit de 400 Mbps y 1.25 Gbps, respectivamente.

\section{Conclusión}

Se ha presentado un nuevo concepto de un ecualizador adaptativo multi-rate para comunicaciones serie de alta velocidad. Se ha simulado un prototipo en tecnología estándar CMOS, capaz de adaptar su respuesta a tasas de transmisión desde 400 Mbps hasta 1.25 Gbps. El circuito opera con una tensión de alimentación de 1 $\mathrm{V}$, lo que demuestra su escalabilidad a tecnologías CMOS nanométrica.

\section{Referencias}

[1]. HYE-YOON, J., HA, K.S., and KIM, L.S.. A datapattern-tolerant adaptive equalizer using the spectrum balancing method. IEEE Transactions on Circuits and Systems II: Express Briefs. 2010, 57(3), 228-232.

[2]. GIMENO, C. GUERRERO, E., CELMA, S. and ALDEA, C. Reliable CMOS adaptive equalizer for short-haul optical networks. Microelectronics Reliability. 2014, 54(1), 110-118.

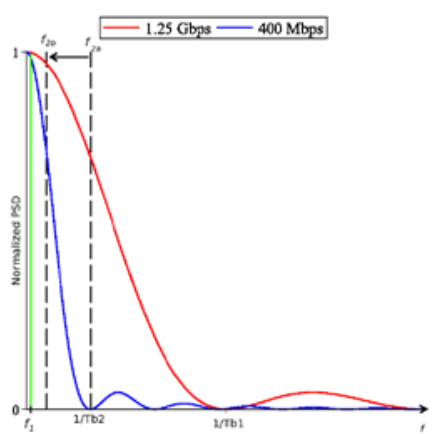

Figura 2. PSD para distintas tasas de transmisión NRZ

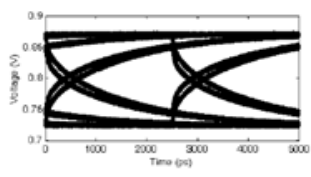

(a)

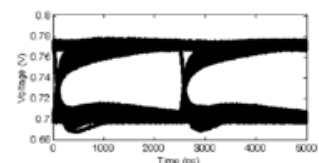

(b)
Figura 4. Diagrama de ojos para 400 Mbps sobre POF de $50 \mathrm{~m}$ (a) antes y (b) después de la ecualización

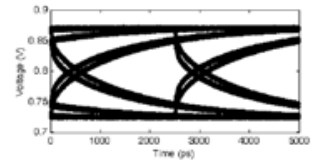

(a)

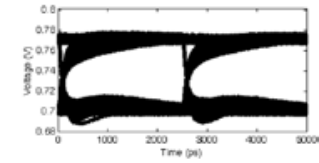

(b)
Figura 5. Diagrama de ojos para 1.25 Gbps sobre POF de $50 \mathrm{~m}$ (a) antes v (b) después de la ecualización 